\title{
Existence and Boundedness of Solutions for Elliptic Equa- tions in General Domains
}

Elhoussine Azrou $\left.\right|^{7}{ }^{1}$, Moussa Khouakhi ${ }^{1}$, Chihab Yazough ${ }^{2}$

${ }^{1}$ Sidi Mohamed Ben Abdellah University, LAMA, FSDM, Fès, Morocco

2 Sidi Mohamed Ben Abdellah University, Mathematics Physics and Computer Science, LSI, FP, Taza, Morocco

Emails:azroul_elhoussine@yahoo.fr,moussa.khouakhi@gmail.com,chihabyazough@gmail.com

\begin{tabular}{l} 
A R T I C L E I N F O \\
\hline Article history: \\
Received: 12 April, 2017 \\
Accepted: 04 May, 2017 \\
Online: 29 December, 2017 \\
Keywords: \\
Unbounded Domains \\
Sobolev Spaces With Variable \\
Exponents \\
Boundedness Of Solutions \\
Strongly Nonlinear Elliptic \\
Equations \\
Existence Results \\
\hline
\end{tabular}

\section{Introduction}

In recent years, there has been an increasing interest in the study of various mathematical problems with variable exponents. These problems are interesting in applications (see [[1], [2]]). For the usual problems when $p$ is constant, there are many results for existence of solutions when the domain is bounded or unbounded. For $p$ variable, when the domain is bounded, on the results of existence of solutions, we refer to [[3], [4], [5]], when the domain is unbounded, results of existence of solutions are rare we can cite for example [[6], [7]].

In the case where $\Omega$ is a bounded, and for $1<p<N$, In [8] authors studied the problem:

$$
\begin{gathered}
-\operatorname{div} a(x, u, \nabla u)=H(x, u, \nabla u)+f-\operatorname{div} g \text { in } D^{\prime}(\Omega), \\
u \in W_{0}^{1, p}(\Omega),
\end{gathered}
$$

where the right hand side is assumed to satisfy:

$$
f \in L^{N / p}(\Omega), g \in\left(L^{N /(p-1)}(\Omega)\right)^{N} .
$$

Under suitable smallness assumptions on $f$ and $g$ they prove the existence of a solution $u$ which satisfies a further regularity.
In [9] in the case of unbounded domains Guowei Dai By variational approach and the theory of the variable exponent Sobolev spaces establish the existence of infinitely many distinct homoclinic radially symmetric solutions whose $W^{1, p(x)}\left(\mathbb{R}^{N}\right)$-norms tend to zero (to infinity, respectively) under weaker hypotheses about nonlinearity at zero (at infinity, respectively).

The principal objective of this paper is to prove the existence and some regularity of solutions of the following $\mathrm{p}(\mathrm{x})$-Laplacian equation in open set $\Omega$ of $\mathbb{R}^{N}$ (possibly of infinite measure):

$$
\begin{aligned}
-\Delta_{p(x)}(u)+\alpha_{0}|u|^{p(x)-2} u & =d(x) \frac{|\nabla u|^{p(x)}}{|u|^{p(x)}+1}+f-\operatorname{div} g(x) \quad \text { in } \Omega, \\
u & \in W_{0}^{1, p(x)}(\Omega),
\end{aligned}
$$

where $\mathrm{p}$ is log-Hölder continuous function such that $1<p_{-} \leq p_{+}<N, \Delta_{p(x)}(u)=\operatorname{div}\left(|\nabla u|^{p(x)-2} \nabla u\right)$ is the $p(x)$-Laplace operator, $\alpha_{0}$ is a positive constant, $d$ is a function in $L^{\infty}(\Omega)$. We assume the following hypotheses on the source terms $f$ and $g$ :

$f: \Omega \rightarrow \mathbb{R}, g: \Omega \rightarrow \mathbb{R}^{N}$ are a measurable function

${ }^{*}$ Corresponding Author: E. Azroul \& azroul_elhoussine@yahoo.fr 
satisfying:

$$
\begin{gathered}
f \in L^{N / p(x)}(\{x \in \Omega: 1<|f(x)|\}), \\
f \in L^{p^{\prime}(x)}(\{x \in \Omega:|f(x)| \leq 1\}) \\
g \in L^{N /(p(x)-1)}\left(\Omega ; \mathbb{R}^{N}\right) \cap L^{p^{\prime}(x)}\left(\Omega ; \mathbb{R}^{N}\right)
\end{gathered}
$$

We will proceed by solving the problem on a sequence $\Omega_{n}$ of bounded sets after that we pass to the limit in the approximating problems by using the a priori estimate (this a priori estimates provide the necessary compactness properties for solutions) from which the desired results are easily inferred. To this aim, we can neither use any embedding theorem between $L^{p(.)}(\Omega)$ nor any argument involving the measure of $\Omega_{n}$, and under suitable assumptions on $f$ and $g$ we prove some regularity of a solutions $u$ of (1). A similar result has been proved in [7] where $\mathrm{p}$ is constant such that $1<p<N$ but in the present setting such an approach cannot be used directly, because of the variability of $p$.

The plan of the paper is the following: In Section 2 we recall some important defnitions and results of variable exponent Lebesgue and Sobolev spaces. In Section 3 we will give the precise assumptions and state the main results. In Section 4 we will define the approximate problems, state the a priori estimates that we want to obtain. In the Sections which follow we will prove strong convergence of $u_{n}$ and their gradients $\nabla u_{n}$. Section 5 is devoted to conclude the proof of the main existence results. Finally, in Section 6, we prove that, if $\mathrm{f}$ and $\mathrm{g}$ have higher integrability, then every solution $u$ of (1) is bounded. More precisely, we will assume that 2) are replaced by:

$$
\begin{gathered}
f \in L^{q(x)}(\{x \in \Omega: 1<|f(x)|\}) \text { for some } q(x)>N / p(x), \\
\quad f \in L^{p^{\prime}(x)}(\{x \in \Omega:|f(x)| \leq 1\}) \\
g \in L^{r(x)}\left(\Omega ; \mathbb{R}^{N}\right) \cap L^{p^{\prime}(x)}\left(\Omega ; \mathbb{R}^{N}\right) \\
\quad \text { for some } r(x)>N /(p(x)-1)
\end{gathered}
$$

\section{Preliminaries}

In order to discuss the problem (1), we need to recall some definitions and basic properties of Lebesgue and Sobolev spaces with variable exponents.

Let $\Omega$ an open bounded set of $\mathbb{R}^{N}$ with $N \geq 2$. We say that a real-valued continuous function $p($.$) is log-$ Hölder continuous in $\Omega$ if:

$$
\begin{aligned}
|p(x)-p(y)| \leq & \frac{C}{|\log | x-y||} \quad \forall x, y \in \bar{\Omega} \\
& \text { such that }|x-y|<\frac{1}{2},
\end{aligned}
$$

We denote:

$$
\begin{array}{r}
C_{+}(\bar{\Omega})=\{\log \text {-Hölder continuous function } \\
\left.p: \bar{\Omega} \rightarrow \mathbb{R} \text { with } 1<p_{-} \leq p_{+}<N\right\},
\end{array}
$$

where:

$$
p_{-}=e s s \min _{x \in \bar{\Omega}} p(x) \quad p_{+}=e s s \sup _{x \in \bar{\Omega}} p(x) .
$$

We define the variable exponent Lebesgue space for $p \in C_{+}(\bar{\Omega})$ by:

$L^{p(x)}(\Omega)=\left\{u: \Omega \rightarrow \mathbb{R}\right.$ measurable $\left.: \int_{\Omega}|u(x)|^{p(x)} d x<\infty\right\}$, the space $L^{p(x)}(\Omega)$ under the norm:

$$
\|u\|_{L^{p(x)}(\Omega)}=\inf \left\{\lambda>0: \int_{\Omega}\left|\frac{u(x)}{\lambda}\right|^{p(x)} d x \leq 1\right\}
$$

is a uniformly convex Banach space, and therefore reflexive.

We denote by $L^{p^{\prime}(x)}(\Omega)$ the conjugate space of $L^{p(x)}(\Omega)$ where $\frac{1}{p(x)}+\frac{1}{p^{\prime}(x)}=1$ (see [10, 11]).

Proposition 1 (Generalized Hölder inequality [10, 11])

(i) For any functions $u \in L^{p(x)}(\Omega)$ and $v \in L^{p^{\prime}(x)}(\Omega)$, we have

$$
\left|\int_{\Omega} u v d x\right| \leq\left(\frac{1}{p_{-}}+\frac{1}{p_{-}^{\prime}}\right)\|u\|_{L^{p(x)}(\Omega)}\|v\|_{L^{p^{\prime}(x)}(\Omega)} .
$$

(ii) For all $p_{1}, p_{2} \in C_{+}(\bar{\Omega})$ such that: $p_{1}(x) \leq p_{2}(x)$ a.e. in $\Omega$, we have: $L^{p_{2}(x)}(\Omega) \hookrightarrow L^{p_{1}(x)}(\Omega)$ and the embedding is continuous.

Proposition $2([\mathbf{1 0}, \mathbf{1 1}])$ If we denote

$$
\rho(u)=\int_{\Omega}|u|^{p(x)} d x \quad \forall u \in L^{p(x)}(\Omega),
$$

then, the following assertions hold

(i) $\|u\|_{L^{p(x)}(\Omega)}<1$ (resp, =1, > 1) if and only if $\rho(u)<1$ (resp, =1, > 1);

(ii) $\|u\|_{L^{p(x)}(\Omega)}>1$ implies $\|u\|_{L^{p(x)}(\Omega)}^{p_{-}} \leq \rho(u) \leq$ $\|u\|_{L^{p(x)}(\Omega)}^{p_{+}}$, and $\|u\|_{L^{p(x)}(\Omega)}<1$ implies $\|u\|_{L^{p(x)}(\Omega)}^{p_{+}} \leq$ $\rho(u) \leq\|u\|_{L^{p(x)}(\Omega)}^{p_{-}} ;$

(iii) $\|u\|_{L^{p(x)}(\Omega)} \rightarrow 0$ if and only if $\rho(u) \rightarrow 0$, and $\|u\|_{L^{p(x)}(\Omega)} \rightarrow \infty$ if and only if $\rho(u) \rightarrow \infty$.

Now, we define the variable exponent Sobolev space by:

$$
W^{1, p(x)}(\Omega)=\left\{u \in L^{p(x)}(\Omega) \text { and }|\nabla u| \in L^{p(x)}(\Omega)\right\},
$$

with the norm:

$\|u\|_{W^{1, p(x)}(\Omega)}=\|u\|_{L^{p(x)}(\Omega)}+\|\nabla u\|_{L^{p(x)}(\Omega)} \quad \forall u \in W^{1, p(x)}(\Omega)$.

We denote by $W_{0}^{1, p(x)}(\Omega)$ the closure of $C_{0}^{\infty}(\Omega)$ in $W^{1, p(x)}(\Omega)$, and we define the Sobolev exponent by $p^{*}(x)=\frac{N p(x)}{N-p(x)}$ for $p(x)<N$.

Proposition $3([10,12])$ (i) If $1<p_{-} \leq p_{+}<\infty$, then the spaces $W^{1, p(x)}(\Omega)$ and $W_{0}^{1, p(x)}(\Omega)$ are separable and reflexive Banach spaces. 
(ii) If $q \in C_{+}(\bar{\Omega})$ and $q(x)<p^{*}(x)$ for any $x \in \Omega$, then the embedding $W_{0}^{1, p(x)}(\Omega) \hookrightarrow \hookrightarrow L^{q(x)}(\Omega)$ is continuous and compact.

(iii) Poincaré inequality: There exists a constant $C>0$, such that:

$$
\|u\|_{L^{p(x)}(\Omega)} \leq C\|\nabla u\|_{L^{p(x)}(\Omega)} \quad \forall u \in W_{0}^{1, p(x)}(\Omega) .
$$

(vi) Sobolev-Poincare inequality: there exists an other constant $C>0$, such that:

$$
\|u\|_{L^{p *(x)}(\Omega)} \leq C\|\nabla u\|_{L^{p(x)}(\Omega)} \quad \forall u \in W_{0}^{1, p(x)}(\Omega) .
$$

The symbol $\rightarrow$ will denote the weak convergence, and the constants $C_{i}, i=1,2, \ldots$ used in each step of proof are independent.

\section{Approximate problems and $\mathrm{A}$ priori estimates}

In this section we will prove the existence result to the approximate problems. Also we will give a uniform estimate for this solutions $u_{n}$.

\section{Approximate problems}

For $k>0$ and $s \in \mathbb{R}$, the truncation function $T_{k}($.$) is$ defined by:

$$
T_{k}(s)= \begin{cases}s & \text { if }|s| \leq k, \\ k \frac{s}{|s|} & \text { if }|s|>k .\end{cases}
$$

Let $\Omega_{n}=\Omega \cap B_{n}(0)$ where $B_{n}(0)$ is the Ball with center 0 and radius $\mathrm{n}$, we consider the approximate problem:

$$
\begin{gathered}
-\Delta_{p(.)}\left(u_{n}\right)+c\left(x, u_{n}\right)=H_{n}\left(x, u_{n}, \nabla u_{n}\right)+f_{n}-\operatorname{div} g_{n} \quad \text { in } \Omega_{n}, \\
u_{n} \in W_{0}^{1, p(.)}\left(\Omega_{n}\right) \cap L^{\infty}\left(\Omega_{n}\right),
\end{gathered}
$$

with $c(x, u)=\alpha_{0}|u|^{p(x)-2} u, H_{n}(x, s, \xi)=T_{n}(H(x, s, \xi))$, $H(x, s, \xi)=d(x) \frac{|\xi|^{p(x)}}{|s|^{p(x)}+1}, \quad f_{n}(x)=T_{n}(f(x))$ and $g_{n}(x)=\frac{g(x)}{1+\frac{1}{n}|g(x)|}$. Let us remark that $\left|H_{n}\right| \leq|H|$, $\left|H_{n}\right| \leq n,\left|f_{n}\right| \leq|f|$ and $\left|g_{n}\right| \leq|g|$.

Lemma 1 ([13]) Let $p$ be a measurable function and $s>0$ such that $s p_{-}>1$ then $\left\|\left.f\right|^{s}\right\|_{L^{p(x)}(\Omega)}=\|f\|_{L^{p(x)}(\Omega)}^{s}$ for every $f$ in $L^{p(x)}(\Omega)$.

Lemma 2 ([3]) Let $a: \Omega \times \mathbb{R} \times \mathbb{R}^{N} \rightarrow \mathbb{R}^{N}$ be a Caratheodory function (measurable with respect to $x$ in $\Omega$ for every $(s, \xi)$ in $\mathbb{R} \times \mathbb{R}^{N}$, and continuous with respect to $(s, \xi)$ in $\mathbb{R} \times \mathbb{R}^{N}$ for almost every $x$ in $\Omega$ ) and let us Assume that:

$$
\begin{gathered}
|a(x, s, \xi)| \leq \beta\left(K(x)+|s|^{p(x)-1}+|\xi|^{p(x)-1}\right), \\
a(x, s, \xi) \xi \geq \alpha|\xi|^{p(x)}, \\
{[a(x, s, \xi)-a(x, s, \bar{\xi})](\xi-\bar{\xi})>0 \text { for all } \xi \neq \bar{\xi} \text { in } \mathbb{R}^{N},}
\end{gathered}
$$

hold, and let $\left(u_{n}\right)_{n}$ be a sequence in $W_{0}^{1, p(x)}(\Omega)$ such that $u_{n} \rightarrow u$ in $W_{0}^{1, p(x)}(\Omega)$ and

$$
\int_{\Omega}\left[a\left(x, u_{n}, \nabla u_{n}\right)-a\left(x, u_{n}, \nabla u\right)\right] \nabla\left(u_{n}-u\right) d x \rightarrow 0,
$$

then $u_{n} \rightarrow u$ in $W_{0}^{1, p(x)}(\Omega)$ for a subsequence.

We define the operator:

$R_{n}: W_{0}^{1, p(x)}\left(\Omega_{n}\right) \rightarrow W^{-1,{ }^{\prime}(x)}\left(\Omega_{n}\right)$, by:

$\left\langle R_{n} u, v\right\rangle=\int_{\Omega_{n}} c(x, u) v-H_{n}(x, u, \nabla u) v d x \quad \forall v \in W_{0}^{1, p(x)}\left(\Omega_{n}\right)$.

by the Hölder inequality we have that:

for all $u, v \in W_{0}^{1, p(x)}\left(\Omega_{n}\right)$,

$$
\begin{aligned}
& \left|\int_{\Omega_{n}} c(x, u) v-H_{n}(x, u, \nabla u) v d x\right| \\
& \leq\left(\frac{1}{p_{-}}+\frac{1}{p_{-}^{\prime}}\right)\left[\|c(x, u)\|_{L^{p^{\prime}(x)}\left(\Omega_{n}\right)}\|v\|_{L^{p(x)}\left(\Omega_{n}\right)}\right. \\
& \left.+\left\|H_{n}(x, u, \nabla u)\right\|_{L^{p^{\prime}(x)}\left(\Omega_{n}\right)}\|v\|_{L^{p(x)}\left(\Omega_{n}\right)}\right] \\
& \leq\left(\frac{1}{p_{-}}+\frac{1}{p_{-}^{\prime}}\right)\left[\left(\int_{\Omega_{n}}\left(|c(x, u)|^{p^{\prime}(x)} d x+1\right)^{\frac{1}{p_{-}^{\prime}}}\right.\right. \\
& +\left(\int_{\Omega_{n}}\left(\left|H_{n}(x, u, \nabla u)\right|^{p^{\prime}(x)} d x+1\right)^{\frac{1}{p_{-}^{\prime}}}\right]\|v\|_{W^{1, p(x)}\left(\Omega_{n}\right)}
\end{aligned}
$$

Then:

$$
\begin{aligned}
& \left|\int_{\Omega_{n}} c(x, u) v+H_{n}(x, u, \nabla u) v d x\right| \\
& \leq\left(\frac{1}{p_{-}}+\frac{1}{p_{-}^{\prime}}\right)\left[\left(\int_{\Omega_{n}}|u|^{p(x)}\right) d x+1\right)^{\frac{1}{p_{-}^{\prime}}} \\
& \left.+\left(\int_{\Omega_{n}} n^{p^{\prime}(x)} d x+1\right)^{\frac{1}{p_{-}^{\prime}}}\right]\|v\|_{W^{1, p(x)}\left(\Omega_{n}\right)} \\
& \leq\left(\frac{1}{p_{-}}+\frac{1}{p_{-}^{\prime}}\right)\left[\left(\int_{\Omega_{n}}|u|^{p(x)}\right) d x+1\right)^{\frac{1}{p_{-}^{\prime}}} \\
& \left.+\left(n^{p_{+}^{\prime}} \cdot \operatorname{meas}\left(\Omega_{n}\right)+1\right)^{\frac{1}{p_{-}^{\prime}}}\right]\|v\|_{W^{1, p(x)}\left(\Omega_{n}\right)} \\
& \leq C_{1}\|v\|_{W^{1, p(x)}\left(\Omega_{n}\right)}
\end{aligned}
$$

Lemma 3 The operator $B_{n}=A+R_{n}$ is pseudo-monotone from $W_{0}^{1, p(x)}\left(\Omega_{n}\right)$ into $W^{-1, p^{\prime}(x)}\left(\Omega_{n}\right)$. Moreover, $B_{n}$ is coercive in the following sense

$$
\begin{gathered}
\frac{\left\langle B_{n} v, v\right\rangle}{\|v\|_{W^{1, p(x)}\left(\Omega_{n}\right)}} \rightarrow+\infty \quad \text { as } \quad\|v\|_{W^{1, p(x)}\left(\Omega_{n}\right)} \rightarrow+\infty \\
\text { for } \quad v \in W_{0}^{1, p(x)}\left(\Omega_{n}\right) .
\end{gathered}
$$

where $A u=-\Delta_{p(x)}(u)$ 
Proof: Using Hölder's inequality we can show that the operator $A$ is bounded, and by using 10 we conclude that $B_{n}$ is bounded. For the coercivity, we have for any $u \in W_{0}^{1, p(x)}\left(\Omega_{n}\right)$,

$$
\begin{aligned}
& \left\langle B_{n} u, u\right\rangle=\langle A u, u\rangle+\left\langle R_{n} u, u\right\rangle \\
& =\int_{\Omega_{n}}|\nabla u|^{p(x)} d x+\int_{\Omega_{n}} c(x, u) u-H_{n}(x, u, \nabla u) u d x \\
& \geq \int_{\Omega}|\nabla u|^{p(x)} d x-C_{1} \cdot\|u\|_{W^{1, p(x)}\left(\Omega_{n}\right)} \quad(\text { using }[10) \\
& \geq\|\nabla u\|_{L^{p(x)}\left(\Omega_{n}\right)}^{\delta^{\prime}}-C_{1} \cdot\|u\|_{W^{1, p(x)}\left(\Omega_{n}\right)} \\
& \geq \alpha^{\prime}\|u\|_{W^{1, p(x)}\left(\Omega_{n}\right)}^{\delta^{\prime}}-C_{1} \cdot\|u\|_{W^{1, p(x)}\left(\Omega_{n}\right)} \\
& \text { (using Poincaré's inequality) }
\end{aligned}
$$

With

$$
\delta^{\prime}= \begin{cases}p_{-} & \text {if }\|\nabla u\|_{L^{p(x)}\left(\Omega_{n}\right)}>1, \\ p_{+} & \text {if }\|\nabla u\|_{L^{p(x)}\left(\Omega_{n}\right)} \leq 1,\end{cases}
$$

Then, we obtain:

$$
\frac{\left\langle B_{n} u, u\right\rangle}{\|u\|_{W^{1, p(x)}\left(\Omega_{n}\right)}} \rightarrow+\infty \quad \text { as }\|u\|_{W^{1, p(x)}\left(\Omega_{n}\right)} \rightarrow+\infty .
$$

It remains now to show that $B_{n}$ is pseudomonotone. Let $\left(u_{k}\right)_{k}$ a sequence in $W_{0}^{1, p(x)}\left(\Omega_{n}\right)$ such that:

$$
\begin{gathered}
u_{k} \rightarrow u \quad \text { in } W_{0}^{1, p(x)}\left(\Omega_{n}\right), \\
B_{n} u_{k} \rightarrow \chi \quad \text { in } W^{-1, p^{\prime}(x)}\left(\Omega_{n}\right), \\
\limsup _{k \rightarrow \infty}\left\langle B_{n} u_{k}, u_{k}\right\rangle \leq\langle\chi, u\rangle .
\end{gathered}
$$

We will prove that:

$$
\chi=B_{n} u \quad \text { and } \quad\left\langle B_{n} u_{k}, u_{k}\right\rangle \rightarrow\langle\chi, u\rangle \quad \text { as } k \rightarrow+\infty .
$$

Firstly, since $W_{0}^{1, p(x)}\left(\Omega_{n}\right) \hookrightarrow \hookrightarrow L^{p(x)}\left(\Omega_{n}\right)$, then $u_{k} \rightarrow u$ in $L^{p(x)}\left(\Omega_{n}\right)$ for a subsequence still denoted by $\left(u_{k}\right)_{k}$.

We have $\left(u_{k}\right)_{k}$ is a bounded sequence in $W_{0}^{1, p(x)}\left(\Omega_{n}\right)$, then $\left(\left|\nabla u_{k}\right|^{p(x)-2} \nabla u_{k}\right)_{k}$ is bounded in $\left(L^{p^{\prime}(x)}\left(\Omega_{n}\right)\right)^{N}$, therefore, there exists a function $\varphi \in\left(L^{p^{\prime}(x)}\left(\Omega_{n}\right)\right)^{N}$ such that:

$$
\left|\nabla u_{k}\right|^{p(x)-2} \nabla u_{k} \rightarrow \varphi \quad \text { in }\left(L^{p^{\prime}(x)}\left(\Omega_{n}\right)\right)^{N} \text { as } k \rightarrow \infty .
$$

Similarly, since $\left(c\left(x, u_{k}\right)-H_{n}\left(x, u_{k}, \nabla u_{k}\right)\right)_{k}$ is bounded in $L^{p^{\prime}(x)}\left(\Omega_{n}\right)$, then there exists a function $\psi_{n} \in L^{p^{\prime}(x)}\left(\Omega_{n}\right)$ such that: $c\left(x, u_{k}\right)-H_{n}\left(x, u_{k}, \nabla u_{k}\right) \rightarrow \psi_{n} \quad$ in $L^{p^{\prime}(x)}\left(\Omega_{n}\right)$ as $k \rightarrow \infty$,

For all $v \in W_{0}^{1, p(x)}\left(\Omega_{n}\right)$, we have:

$$
\begin{aligned}
\langle\chi, v\rangle & =\lim _{k \rightarrow \infty}\left\langle B_{n} u_{k}, v\right\rangle \\
& =\lim _{k \rightarrow \infty} \int_{\Omega_{n}}\left|\nabla u_{k}\right|^{p(x)-2} \nabla u_{k} \nabla v d x \\
& +\lim _{k \rightarrow \infty} \int_{\Omega_{n}}\left(c\left(x, u_{k}\right)-H_{n}\left(x, u_{k}, \nabla u_{k}\right)\right) v d x \\
& =\int_{\Omega_{n}} \varphi \nabla v d x+\int_{\Omega_{n}} \psi_{n} v d x .
\end{aligned}
$$

Using 11 and (14, we obtain:

$$
\begin{aligned}
& \limsup _{k \rightarrow \infty}\left\langle B_{n}\left(u_{k}\right), u_{k}\right\rangle \\
& =\limsup _{k \rightarrow \infty}\left\{\int_{\Omega_{n}}\left|\nabla u_{k}\right|^{p(x)} d x+\int_{\Omega_{n}}\left(c\left(x, u_{k}\right)-H_{n}\left(x, u_{k}, \nabla u_{k}\right)\right) u_{k} d x\right\} \\
& \leq \int_{\Omega} \varphi \nabla u d x+\int_{\Omega} \psi_{n} u d x
\end{aligned}
$$

Thanks to 13, we have:

$$
\int_{\Omega_{n}}\left(c\left(x, u_{k}\right)-H_{n}\left(x, u_{k}, \nabla u_{k}\right)\right) u_{k} d x \rightarrow \int_{\Omega_{n}} \psi_{n} u d x
$$

Therefore,

$$
\limsup _{k \rightarrow \infty} \int_{\Omega_{n}}\left|\nabla u_{k}\right|^{p(x)} d x \leq \int_{\Omega_{n}} \varphi \nabla u d x .
$$

On the other hand, we have:

$$
\int_{\Omega_{n}}\left(\left|\nabla u_{k}\right|^{p(x)-2} \nabla u_{k}-|\nabla u|^{p(x)-2} \nabla u\right)\left(\nabla u_{k}-\nabla u\right) d x \geq 0,
$$

Then

$$
\begin{aligned}
\int_{\Omega_{n}}\left|\nabla u_{k}\right|^{p(x)} d x & \geq-\int_{\Omega_{n}}|\nabla u|^{p(x)} d x+\int_{\Omega_{n}}\left|\nabla u_{k}\right|^{p(x)-2} \nabla u_{k} \nabla u d x \\
& +\int_{\Omega_{n}}|\nabla u|^{p(x)-2} \nabla u \nabla u_{k} d x
\end{aligned}
$$

and by 12 , we get:

$$
\liminf _{k \rightarrow \infty} \int_{\Omega_{n}}\left|\nabla u_{k}\right|^{p(x)} d x \geq \int_{\Omega_{n}} \varphi \nabla u d x,
$$

this implies, thanks to (17), that:

$$
\lim _{k \rightarrow \infty} \int_{\Omega_{n}}\left|\nabla u_{k}\right|^{p(x)} d x=\int_{\Omega_{n}} \varphi \nabla u d x .
$$

By combining 14, (16) and 19, we deduce that:

$$
\left\langle B_{n} u_{k}, u_{k}\right\rangle \rightarrow\langle\chi, u\rangle \quad \text { as } k \rightarrow+\infty .
$$

Now, by 19 we can obtain:

$\left.\lim _{k \rightarrow+\infty} \int_{\Omega_{n}}\left(\left|\nabla u_{k}\right|^{p(x)-2} \nabla u_{k}-|\nabla u|^{p(x)-2} \nabla u\right)\right)\left(\nabla u_{k}-\nabla u\right) d x=0$,

In view of the Lemma 2, we obtain:

$$
u_{k} \rightarrow u, \quad W_{0}^{1, p(x)}\left(\Omega_{n}\right), \quad \nabla u_{k} \rightarrow \nabla u \quad \text { a.e. in } \Omega_{n},
$$

then

$$
\left|\nabla u_{k}\right|^{p(x)-2} \nabla u_{k} \rightarrow|\nabla u|^{p(x)-2} \nabla u \quad \text { in }\left(L^{p^{\prime}(x)}\left(\Omega_{n}\right)\right)^{N},
$$

and

$c\left(x, u_{k}\right)-H_{n}\left(x, u_{k}, \nabla u_{k}\right) \rightarrow c(x, u)-H_{n}(x, u, \nabla u) \quad$ in $L^{p^{\prime}(x)}\left(\Omega_{n}\right)$,

we deduce that $\chi=B_{n} u$, which completes the proof.

By Lemma 3, we deduce that there exists at least one weak solution $u_{n} \in W_{0}^{1, p(x)}\left(\Omega_{n}\right)$ of the problem (5), (cf. [14]). 


\section{A priori estimates}

Proposition 4 Assuming that $p(.) \in C_{+}(\bar{\Omega})$ holds, and let $u_{n}$ be any solution of (5). Then for every $\lambda>0$ there exists a positive constant $C=C\left(N, p, \alpha_{0}, d, f, g, \lambda\right)$ such that:

$$
\left\|e^{\lambda\left|u_{n}\right|}-1\right\|_{W_{0}^{1, p(x)}\left(\Omega_{n}\right)} \leq C .
$$

Remark 1 The previous estimate yields an estimate for the functions $e^{\lambda\left|u_{n}\right|}$ in $L_{l o c}^{r(x)}(\Omega)$ for every $r \in[1,+\infty)$, every $\lambda>0$ and every set $\Omega_{0} \subset \subset \Omega$, one has

$$
\left\|e^{\left|u_{n}\right|}\right\|_{L^{r(x)}\left(\Omega_{0}\right)} \leq C\left(r_{\mp}, \lambda, \Omega_{0}\right)
$$

Proof:

For simplicity of notation we will always omit the index $n$ of the sequence. We take $\varphi\left(G_{k}(u)\right)$ as test function in (5), where

$$
G_{k}(s)=s-T_{k}(s)= \begin{cases}s-k & \text { if } s>k, \\ 0 & \text { if }|s| \leq k, \\ s+k & \text { if } s<-k .\end{cases}
$$$$
\text { and } \varphi(s)=\left(e^{\lambda|s|}-1\right) \operatorname{sign}(s) \text {. }
$$

we have:

$$
\begin{aligned}
& \int_{\Omega}\left|\nabla G_{k}(u)\right|^{p(x)} \varphi^{\prime}\left(G_{k}(u)\right)+\alpha_{0} \int_{\Omega}|u|^{p(x)-1}\left|\varphi\left(G_{k}(u)\right)\right|, \\
& \leq d \int_{\Omega}\left|\nabla G_{k}(u)\right|^{p(x)}\left|\varphi\left(G_{k}(u)\right)\right|+\int_{\Omega} \mid f \| \varphi\left(G_{k}(u)\right) \\
& +\int_{\Omega}|g| \nabla G_{k}(u) \mid \varphi^{\prime}\left(G_{k}(u)\right) \\
& =I+J+K,
\end{aligned}
$$

For every $s$ in $\mathbb{R}$ and if $\lambda$ satisfies:

$$
\lambda \geq 8 d
$$

we have:

$$
d|\varphi(s)| \leq \frac{1}{8} \varphi^{\prime}(s)
$$

then

$$
I \leq \frac{1}{8} \int_{\Omega}\left|\nabla G_{k}(u)\right|^{p(x)} \varphi^{\prime}\left(G_{k}(u)\right)
$$

Before estimating $J$, we remark that:

$$
\int_{\Omega}\left|\nabla G_{k}(u)\right|^{p(x)} \varphi^{\prime}\left(G_{k}(u)\right)=\int_{\Omega}\left|\nabla \Psi\left(G_{k}(u)\right)\right|^{p(x)}
$$

where

$$
\Psi(s)=\int_{0}^{|s|}\left(\varphi^{\prime}(t)\right)^{\frac{1}{p(x)}} d t=\frac{p(x)}{\lambda^{\frac{1}{p^{\prime}(x)}}}\left(e^{\frac{\lambda|s|}{p(x)}}-1\right)
$$

Moreover, we observe that there exists a positive constant $c_{2}=c_{2}(p, \lambda)$ such that

$$
|\varphi(s)| \leq c_{2}(\Psi(s))^{p(x)} \text { for every s such that }|s| \geq 1
$$

Now let us observe that $p$ is a continuous variable exponent on $\bar{\Omega}$ then there exists a constant $\delta>0$ such that:

$\max _{y \in \overline{B(x, \delta) \cap \Omega}} \frac{N-p(y)}{N p(y)} \leq \min _{y \in \overline{B(x, \delta) \cap \Omega}} \frac{p(y)(N-p(y))}{N p(y)} \quad$ for all $x \in \Omega$.

while $\bar{\Omega}$ is compact then we can cover it with a finite number of balls $B_{i}$ for $i=1, \ldots, m$ from 29] we can deduce the pointwise estimate:

$$
1<p_{-, i} \leq p_{+, i} \leq \frac{p_{-, i}^{2} N}{N-p_{-, i}+p_{-, i}^{2}}<N .
$$

is satisfy for all $i=1, \ldots, m$.

$p_{-, i}, p_{+, i}$ denote the local minimum and the local maximum of $p$ on $\overline{B_{i} \cap \Omega}$ respectively

Estimation of the integral $J$ :

Let $H \geq 1$ be a constant that we will chose later. We can estimate $J$ by splitting it as follows:

$$
\begin{aligned}
J & =\sum_{i=0}^{m}\left[\int_{B_{i} \cap\left\{|f|>H,\left|G_{k}(u)\right| \geq 1\right\}}\left|f \| \varphi\left(G_{k}(u)\right)\right|\right] \\
& +\int_{\left\{|f|>H,\left|G_{k}(u)\right|<1\right\}}\left|f\left\|\varphi\left(G_{k}(u)\right)\left|+\int_{\{|f| \leq H\}}\right| f\right\| \varphi\left(G_{k}(u)\right)\right| \\
& =J_{1}+J_{2}+J_{3}
\end{aligned}
$$

By 28) $J_{1}$, can be estimated as follows

$$
J_{1} \leq c_{2} \sum_{i=0}^{m}\left[\int_{B_{i} \cap\left\{|f|>H,\left|G_{k}(u)\right| \geq 1\right\}}|f| \Psi\left(G_{k}(u)\right)^{p(x)}\right]
$$

Let $\epsilon$ a positive constant to be chosen later. Using Young, Sobolev's embedding and Lemma 1 we have:

$$
\begin{aligned}
J_{1} & \leq C \int_{\{|f|>H\}}|f|^{\frac{\epsilon N}{\epsilon N+p(x)-N}}+\frac{1}{8} \sum_{i=0}^{m}\left\|\nabla \Psi\left(G_{k}(u)\right)\right\|_{L^{p(x)}\left(B_{i}\right)}^{\epsilon p_{+, i}^{*}} \\
& \leq C \int_{\{|f|>H\}}|f|^{\frac{\epsilon N}{\epsilon N+p(x)-N}}+\frac{1}{8} \sum_{i=0}^{m}\left[\int_{B_{i}}\left|\nabla \Psi\left(G_{k}(u)\right)\right|^{p(x)}\right]^{\frac{\epsilon+, i}{p+, i}}
\end{aligned}
$$

where $p^{*}(x)=\frac{N p(x)}{N-p(x)}$ and $p_{+, i}^{*}=\frac{N p_{+, i}}{N-p_{+, i}}$ since 30 we can choose $\epsilon$ such that:

$$
\frac{N-p_{-, i}}{N p_{-, i}} \leq \epsilon \leq \frac{p_{-, i}}{p_{+, i}^{*}}
$$

Then using 31 and 26) we obtain that :

$$
J_{1} \leq C \int_{\{|f|>H\}}|f|^{\frac{N}{p(x)}}+\frac{1}{8} \int_{\Omega}\left|\nabla G_{k}(u)\right|^{p(x)} \varphi^{\prime}\left(G_{k}(u)\right.
$$

Remark 2 The cases where $\left\|\Psi\left(G_{k}(u)\right)\right\|_{L^{p *(x)}(\Omega)} \leq 1$ or $\left\|\nabla \Psi\left(G_{k}(u)\right)\right\|_{L^{p(x)}(\Omega)} \leq 1$ are easy to see that $J_{1} \leq C(C$ depend on the data of the problem) 
On the other hand

$$
J_{2} \leq \varphi(1) \int_{\{|f|>H\}}|f| \leq \frac{\varphi(1)}{H^{\frac{N-p_{+}}{p_{+}}}} \int_{\{|f|>H\}}|f|^{\frac{N}{p(x)}}
$$

Finally, if we choose $\mathrm{k}$ sufficiently large such that:

$$
\alpha_{0} k^{p_{-}-1} \geq 4 H
$$

We obtain:

$$
\begin{aligned}
J_{3} & \leq \frac{\alpha_{0}}{4} \int_{\Omega} k^{p(x)-1}\left|\varphi\left(G_{k}(u)\right)\right| \\
& \leq \frac{\alpha_{0}}{4} \int_{\Omega}|u|^{p(x)-1}\left|\varphi\left(G_{k}(u)\right)\right|
\end{aligned}
$$

Estimation of the integral $K$ :

Thanks to Young's inequality, we have:

$$
\begin{aligned}
K \leq & \frac{1}{8} \int_{\Omega}\left|\nabla G_{k}(u)\right|^{p(x)} \varphi^{\prime}\left(G_{k}(u)\right) \\
& +C_{4} \int_{\Omega}|g|^{p^{\prime}(x)} \varphi^{\prime}\left(G_{k}(u)\right), \\
& =K_{1}+K_{2}
\end{aligned}
$$

The integral $K_{2}$ can be estimated as follows:

$$
\begin{aligned}
K_{2} & \leq C_{4} \int_{\Omega}|g|^{p^{\prime}(x)} \varphi^{\prime}\left(G_{k}(u)\right), \\
& \leq C_{4} \lambda e^{\lambda} \int_{\left\{\left|G_{k}(u)\right|<1\right\}}|g|^{p^{\prime}(x)} \\
& +C_{4} \sum_{i=0}^{m}\left[\int_{B_{i} \cap\left\{|g|>1,\left|G_{k}(u)\right|>1\right\}}|g|^{p^{\prime}(x)} \varphi^{\prime}\left(G_{k}(u)\right)\right] \\
& +C_{4} \int_{\left\{|g| \leq 1,\left|G_{k}(u)\right|>1\right\}} \varphi^{\prime}\left(G_{k}(u)\right) \\
& =K_{2,1}+K_{2,2}+K_{2,3}
\end{aligned}
$$

Since $\varphi^{\prime}(s) \leq C_{5}(\Psi(s))^{p(x)}$ for every $s$ such that $|s| \geq 1$, we have:

$$
K_{2,2} \leq C_{6} \sum_{i=0}^{m}\left[\int_{B_{i} \cap\left\{|g|>1,\left|G_{k}(u)\right|>1\right\}}|g|^{p^{\prime}(x)} \Psi\left(G_{k}(u)\right)^{p(x)}\right]
$$

Let $\epsilon$ be a positive constant such that 31). Using Young, Sobolev's embedding and Lemma 1 we have:

$K_{2,2} \leq C_{7} \int_{\{|g|>1\}}|g|^{\frac{\epsilon N p^{\prime}(x)}{\epsilon N+p(x)-N}}+\frac{1}{8} \sum_{i=0}^{m}\left\|\nabla \Psi\left(G_{k}(u)\right)\right\|_{L^{p(x)}\left(B_{i}\right)}^{\epsilon p_{+, i}^{*}}$ $\leq C_{7} \int_{\{|g|>1\}}|g|^{\frac{\epsilon N p^{\prime}(x)}{\epsilon N+p(x)-N}}+\frac{1}{8} \sum_{i=0}^{m}\left[\int_{B_{i}}\left|\nabla \Psi\left(G_{k}(u)\right)\right|^{p(x)}\right]^{\frac{\epsilon p_{,+i}^{*}}{p_{-, i}}}$

Then using 231 and 26) we obtain that:

$$
K_{2,2} \leq C_{7} \int_{\{|g|>1\}}|g|^{\frac{N}{p(x)-1}}+\frac{1}{8} \int_{\Omega}\left|\nabla G_{k}(u)\right|^{p(x)} \varphi^{\prime}\left(G_{k}(u)\right.
$$

The same as before in the cases where $\left\|\Psi\left(G_{k}(u)\right)\right\|_{L^{p *(x)}(\Omega)} \leq 1$ or $\left\|\nabla \Psi\left(G_{k}(u)\right)\right\|_{L^{p(x)}(\Omega)} \leq 1$ it is easy to check that $K_{2,2} \leq C$

Finally, using inequality

$$
\varphi^{\prime}(s) \leq C_{8}|\varphi(s)|, \quad \text { for every s such that }|s| \geq 1
$$

and choosing $k=k\left(p_{-}, \alpha_{0}, \lambda\right)$ sufficiently large such that:

$$
\alpha_{0} k^{p_{-}-1} \geq 4 C_{4}
$$

we obtain:

$$
\begin{aligned}
K_{2,3} & \leq \frac{\alpha_{0}}{4} \int_{\Omega} k^{p(x)-1}\left|\varphi\left(G_{k}(u)\right)\right| \\
& \leq \frac{\alpha_{0}}{4} \int_{\Omega}|u|^{p(x)-1}\left|\varphi\left(G_{k}(u)\right)\right|
\end{aligned}
$$

Putting all the inequalities (22), (25), (32), (33), (35), (38), 41, (37) and (36) together, we get an estimate in $W_{0}^{1, p(x)}(\Omega)$ for $G_{k}(u)$,when $\mathrm{k}$ is large enough:

$$
\begin{aligned}
& \frac{1}{2} \int_{\Omega}\left|\nabla G_{k}(u)\right|^{p(x)} \varphi^{\prime}\left(G_{k}(u)\right)+\frac{\alpha_{0}}{2} \int_{\Omega}|u|^{p(x)-1}\left|\varphi\left(G_{k}(u)\right)\right| \\
& \leq C \int_{\{|f|>H\}}|f|^{\frac{N}{p(x)}}+\frac{\varphi(1)}{H^{\frac{N-p_{+}}{p_{+}}}} \int_{\{|f|>H\}}|f|^{\frac{N}{p(x)}} \\
& +C_{4} \lambda e^{\lambda} \int_{\Omega}|g|^{p^{\prime}(x)}+C_{7} \int_{\Omega}|g|^{\frac{N}{p(x)-1}} \\
& =C_{9}\left(N, p_{-}, p_{+}, \alpha_{0}, f, g, \lambda\right)
\end{aligned}
$$

For every $\lambda, k$ satisfying (23), (34), (40) and for every $H \geq 1$. We fix now $\lambda$ and $\mathrm{k}$ such that $(42$ holds. As before, If we take $\varphi\left(T_{k}(u)\right)$ as a test function in (5) we obtain:

$$
\begin{aligned}
& \int_{\Omega}\left|\nabla T_{k}(u)\right|^{p(x)} \varphi^{\prime}\left(T_{k}(u)\right)+\alpha_{0} \int_{\Omega}|u|^{p(x)-1}\left|\varphi\left(T_{k}(u)\right)\right|, \\
& \leq d \int_{\Omega}\left|\nabla T_{k}(u)\right|^{p(x)}\left|\varphi\left(T_{k}(u)\right)\right|+d \varphi(k) \int_{\Omega}\left|\nabla G_{k}(u)\right|^{p(x)} \mid \\
& +\int_{\Omega}|f|\left|\varphi\left(T_{k}(u)\right)\right|+\int_{\Omega}|g| \nabla T_{k}(u) \mid \varphi^{\prime}\left(T_{k}(u)\right) \\
& =L_{1}+L_{2}+L_{3}+L_{4},
\end{aligned}
$$

Using 24, we have:

By 442 ,

$$
L_{1} \leq \frac{1}{4} \int_{\Omega}\left|\nabla T_{k}(u)\right|^{p(x)} \varphi^{\prime}\left(T_{k}(u)\right.
$$

$$
L_{2} \leq C_{10}\left(N, p_{-}, p_{+}, \alpha_{0}, f, g, \lambda\right)
$$

Remark 3 if meas $(\Omega)$ is finite or if $f \in L^{1}(\Omega)$ it is easy to estimate the integral $L_{3}$

In general case, let $\epsilon$ be a positive constant to be chosen later, we write

$$
\begin{aligned}
L_{3} & \leq \varphi(k) \int_{\{|f|>1\}}|f|+\int_{\{|f| \leq 1\}}\left|f \| \varphi\left(T_{k}(u)\right)\right| \\
& \leq \varphi(k) \int_{\{|f|>1\}}|f|+\epsilon \int_{\Omega}\left|\varphi\left(T_{k}(u)\right)\right|^{p(x)} \\
& +c\left(\epsilon, p_{-}^{\prime}\right) \int_{\{|f| \leq 1\}}|f|^{p^{\prime}(x)}
\end{aligned}
$$


Since

$$
\left|\varphi\left(T_{k}(u)\right)\right|^{p(x)} \leq C_{11}\left(\lambda, p_{+}, p_{-}, k\right)\left|\varphi\left(T_{k}(u)\right)\right||u|^{p(x)-1},
$$

choosing $\epsilon$ such that $\epsilon C_{11} \leq \frac{\alpha_{0}}{2}$,we have:

$$
L_{3} \leq \frac{\alpha_{0}}{2} \int_{\Omega}|u|^{p(x)-1}\left|\varphi\left(T_{k}(u)\right)\right|+C_{12}\left(\alpha_{0}, f, \lambda, p_{+}, p_{-}, k\right)
$$

Finally, one has

$$
L_{4} \leq \frac{1}{4} \int_{\Omega}\left|\nabla T_{k}(u)\right|^{p(x)} \varphi^{\prime}\left(T_{k}(u)+C_{13}\left(\alpha_{0}, \lambda, p_{-}^{\prime}, g, p_{-}, k\right)\right.
$$

In conclusion, putting all the estimations ( 43 - 47$)$ together, we get:

$$
\begin{aligned}
\frac{1}{2} \int_{\Omega}\left|\nabla T_{k}(u)\right|^{p(x)} & \varphi^{\prime}\left(T_{k}(u)\right)+\frac{\alpha_{0}}{2} \int_{\Omega}|u|^{p(x)-1}\left|\varphi\left(T_{k}(u)\right)\right| \\
& \leq C_{14}\left(N, p_{-}, p_{+}, \alpha_{0}, f, g, \lambda\right)
\end{aligned}
$$

In view of (42) and (48), we have:

$\int_{\{|u| \leq k\}}|\nabla u|^{p(x)} e^{\lambda|u|} \leq C_{15}, \quad \int_{\{|u|>k\}}|\nabla u|^{p(x)} e^{\lambda(|u|-k)} \leq C_{15}$

For every $\lambda, k$ large enough (see (23), (34) and (40)), where $C_{15}$ depends on $\lambda, k$ and the data. Since

$$
\begin{aligned}
\int_{\Omega}|\nabla u|^{p(x)} e^{\lambda|u|} & =\int_{\{|u| \leq k\}}|\nabla u|^{p(x)} e^{\lambda|u|} \\
& +e^{\lambda k} \int_{\{|u|>k\}}|\nabla u|^{p(x)} e^{\lambda(|u|-k)} \\
& \leq C_{16}
\end{aligned}
$$

If we fix the value of $k$ (depending on $\lambda$ ), we obtain an estimate of $\left|\nabla\left(e^{\lambda|u|}-1\right)\right|$ in $L^{p(x)}(\Omega)$ (depending on ג). This implies, by Sobolev's embedding, that:

$$
\int_{\Omega}\left(e^{\lambda|u|}-1\right)^{p *(x)} \leq C_{17}
$$

For every $\lambda$ such that 23 , where $C_{17}$ depends on $\lambda$ and on the data of the problem. Note that $(49)$ does not imply an estimate in $L^{p(x)}(\Omega)$ for $e^{\lambda|u|}-1$, since meas $(\Omega)$ may be infinite. To obtain such an estimate, we have to combine (48) and 49), since, for every $k>0$, one has the inequalities

$$
\begin{gathered}
\int_{\{|u| \leq k\}}\left(e^{\lambda|u|}-1\right)^{p(x)} \leq C_{19} \int_{\Omega}|u|^{p(x)-1} \mid \varphi\left(T_{k}(u) \mid,\right. \\
\int_{\{|u|>k\}}\left(e^{\lambda|u|}-1\right)^{p(x)} \leq C_{20} \int_{\Omega}\left(e^{\lambda|u|}-1\right)^{p *(x)},
\end{gathered}
$$

Therefore, if $k=k(\lambda)$ is such that 48 holds, we can write

$$
\begin{aligned}
& \int_{\Omega}\left(e^{\lambda|u|}-1\right)^{p(x)} \\
& =\int_{\{|u| \leq k\}}\left(e^{\lambda|u|}-1\right)^{p(x)}+\int_{\{|u|>k\}}\left(e^{\lambda|u|}-1\right)^{p(x)} \leq C_{21}
\end{aligned}
$$

where $C_{21}$ depends on $\lambda$ and the data of the problem.

\section{Main results}

In this section we will prove the main result of this paper. Let $\left\{u_{n}\right\}$ be any sequence of solutions of problem (5), we extend them to zero in $\Omega \backslash \Omega_{n}$. By (20), there exist a subsequence (still denoted by $u_{n}$ ) and a function $u \in W_{0}^{1, p(x)}(\Omega)$ such that $u_{n} \rightarrow u$ weakly in $W_{0}^{1, p(x)}(\Omega)$.

Theorem 1 There exists at least one solution $u$ of 11 ; which is such that

$$
\begin{aligned}
\int_{\Omega}|\nabla u|^{p(x)-2} \nabla u \nabla \psi d x & +\int_{\Omega} c(x, u) \psi d x+\int_{\Omega} H(x, u, \nabla u) \psi d x \\
& =\int_{\Omega} f \psi d x-\int_{\Omega} g \nabla \psi d x
\end{aligned}
$$

for every function $\psi \in W_{0}^{1, p(x)}(\Omega) \cap L^{\infty}(\Omega)$. Moreover $u$ satisfies

$$
e^{\lambda|u|}-1 \in W_{0}^{1, p(x)}(\Omega)
$$

for every $\lambda \geq 0$.

The proof will be made in three steps.

\section{Step 1: An estimate for $\int_{\Omega}\left|\nabla G_{k}\left(u_{n}\right)\right|^{p(x)}$}

In view of 42 we have:

$$
\begin{aligned}
& \int_{\Omega}\left|\nabla G_{k}\left(u_{n}\right)\right|^{p(x)} \\
& \leq \frac{2 C}{\lambda} \int_{\{|f|>H\}}|f|^{\frac{N}{p(x)}}+\frac{2 \varphi(1)}{\lambda H^{\frac{N-p_{+}}{p_{+}}}} \int_{\{|f|>H\}}|f|^{\frac{N}{p(x)}}+\frac{2 C_{4} \lambda e^{\lambda}}{\lambda} \int_{\Omega}|g|^{p^{\prime}(x)} \\
& +\frac{2 C_{7}}{\lambda} \int_{\Omega}|g|^{\frac{N}{p(x)-1}}
\end{aligned}
$$

If $\eta$ is an arbitrary positive number, let us choose $H$ such that the right-hand side of (53) is smaller than $\eta$. It follows that, for every $k$ satisfying 34,240 , every $\lambda$ satisfying 23, and every $n \in \mathbb{N}$

$$
\int_{\Omega}\left|\nabla G_{k}\left(u_{n}\right)\right|^{p(x)} \leq \eta
$$

which proves:

$$
\sup _{n} \int_{\Omega}\left|\nabla G_{k}\left(u_{n}\right)\right|^{p(x)} \rightarrow 0 \quad \text { as } \quad k \rightarrow \infty
$$

\section{Step 2: Strong convergence of $\nabla T_{k}\left(u_{n}\right)$}

In this step, we will fix $k>0$ and prove that $\nabla T_{k}\left(u_{n}\right) \rightarrow$ $\nabla T_{k}(u)$ strongly in $L^{p(x)}\left(\Omega_{0} ; \mathbb{R}^{N}\right)$ as $n \rightarrow \infty$; for $k$ fixed. In order to prove this result we define:

$$
z_{n}(x)=T_{k}\left(u_{n}\right)-T_{k}(u)
$$

and we choose $\psi$ a cut-off function such that

$$
\psi \in C_{0}^{\infty}(\Omega), \quad 0 \leq \psi \leq 1, \quad \psi=0 \quad \text { in } \quad \Omega_{0}
$$


Let us take:

$$
v=\varphi\left(z_{n}\right) e^{\delta\left|u_{n}\right|} \psi
$$

as a test function in (5), where $\lambda$ and $\delta$ are a positive constant to be chosen later, we obtain:

$$
\begin{aligned}
& A_{n}+B_{n}=\int_{\Omega}\left|\nabla u_{n}\right|^{p(x)-2} \nabla u_{n} \nabla z_{n} \varphi^{\prime}\left(z_{n}\right) e^{\delta\left|u_{n}\right|} \psi \\
& +\int_{\Omega} c\left(u_{n}\right) \varphi\left(z_{n}\right) e^{\delta\left|u_{n}\right|} \psi \\
& \leq d \int_{\Omega}\left|\nabla u_{n}\right|^{p(x)}\left|\varphi\left(z_{n}\right)\right| e^{\delta\left|u_{n}\right|} \psi+\int_{\Omega}|f|\left|\varphi\left(z_{n}\right)\right| e^{\delta\left|u_{n}\right|} \psi \\
& -\delta \int_{\Omega}\left|\nabla u_{n}\right|^{p(x)} \varphi\left(z_{n}\right) e^{\delta\left|u_{n}\right|} \operatorname{sign}\left(u_{n}\right) \psi \\
& +\int_{\Omega}\left|\nabla u_{n}\right|^{p(x)-1}\left|\nabla \psi \| \varphi\left(z_{n}\right)\right| e^{\delta\left|u_{n}\right|} \\
& +\int_{\Omega}\left|g \| \nabla z_{n}\right| \varphi^{\prime}\left(z_{n}\right) e^{\delta\left|u_{n}\right|} \psi \\
& +\delta \int_{\Omega}|g|\left|\nabla u_{n}\left\|\varphi\left(z_{n}\right)\left|e^{\delta\left|u_{n}\right|} \psi+\int_{\Omega}\right| g|| \nabla \psi\right\| \varphi\left(z_{n}\right)\right| e^{\delta\left|u_{n}\right|} \\
& =C_{n}+D_{n}+E_{n}+F_{n}+G_{n}+H_{n}+L_{n}
\end{aligned}
$$

Splitting $\Omega$ into $\Omega=\left\{\left|u_{n}\right| \leq k\right\} \cup\left\{\left|u_{n}\right|>k\right\}$ we can write:

$A_{n}=\int_{\left\{\left|u_{n}\right| \leq k\right\}}\left|\nabla T_{k}\left(u_{n}\right)\right|^{p(x)-2} \nabla T_{k}\left(u_{n}\right) \nabla z_{n} \varphi^{\prime}\left(z_{n}\right) e^{\delta\left|T_{k}\left(u_{n}\right)\right|} \psi$

$+\int_{\left\{\left|u_{n}\right|>k\right\}}\left|\nabla u_{n}\right|^{p(x)-2} \nabla u_{n} \nabla z_{n} \varphi^{\prime}\left(z_{n}\right) e^{\delta\left|u_{n}\right|} \psi$

$=\int_{\left\{\left|u_{n}\right| \leq k\right\}}\left[\left|\nabla T_{k}\left(u_{n}\right)\right|^{p(x)-2} \nabla T_{k}\left(u_{n}\right)-\left|\nabla T_{k}(u)\right|^{p(x)-2} \nabla T_{k}(u)\right]$

$* \nabla z_{n} \varphi^{\prime}\left(z_{n}\right) e^{\delta\left|T_{k}\left(u_{n}\right)\right|} \psi$

$+\int_{\left\{\left|u_{n}\right| \leq k\right\}}\left|\nabla T_{k}(u)\right|^{p(x)-2} \nabla T_{k}(u) \nabla z_{n} \varphi^{\prime}\left(z_{n}\right) e^{\delta\left|T_{k}\left(u_{n}\right)\right|} \psi$

$+\int_{\left\{\left|u_{n}\right|>k\right\}}\left|\nabla u_{n}\right|^{p(x)-2} \nabla u_{n} \nabla z_{n} \varphi^{\prime}\left(z_{n}\right) e^{\delta\left|u_{n}\right|} \psi$

$=A_{1, n}+A_{2, n}+A_{3, n}$

since

$$
\begin{aligned}
& \left|\nabla T_{k}(u)\right|^{p(x)-2} \nabla T_{k}(u) \varphi^{\prime}\left(z_{n}\right) e^{\delta\left|T_{k}\left(u_{n}\right)\right|} \psi \chi_{\left\{\left|u_{n}\right| \leq k\right\}} \\
& \quad \rightarrow\left|\nabla T_{k}(u)\right|^{p(x)-2} \nabla T_{k}(u) \varphi^{\prime}(0) e^{\delta\left|T_{k}(u)\right|} \psi \chi_{\{|u| \leq k\}}
\end{aligned}
$$

almost everywhere in $\Omega$ (on the set where $|u(x)|=k$ we have $\left.\left|\nabla T_{k}(u)\right|^{p(x)-2} \nabla T_{k}(u)=0\right)$ and

$$
\begin{array}{r}
\|\left.\nabla T_{k}(u)\right|^{p(x)-2} \nabla T_{k}(u) \varphi^{\prime}\left(z_{n}\right) e^{\delta\left|T_{k}\left(u_{n}\right)\right|} \psi \chi_{\left\{\left|u_{n}\right| \leq k\right\}} \mid \\
\leq|\nabla u|^{p(x)-1} \varphi^{\prime}(2 k) e^{\delta k} \psi
\end{array}
$$

which is a fixed function in $L^{p^{\prime}(x)}(\Omega)$. Therefore by Lebesgue's theorem we have

$$
\begin{aligned}
\left|\nabla T_{k}(u)\right|^{p(x)-2} \nabla T_{k}(u) \varphi^{\prime}\left(z_{n}\right) e^{\delta\left|T_{k}\left(u_{n}\right)\right|} \psi \chi_{\left\{\left|u_{n}\right| \leq k\right\}} \\
\quad \rightarrow\left|\nabla T_{k}(u)\right|^{p(x)-2} \nabla T_{k}(u) \varphi^{\prime}(0) e^{\delta\left|T_{k}(u)\right|} \psi \chi_{\{|u| \leq k\}}
\end{aligned}
$$

strongly in $L^{p^{\prime}(x)}(\Omega)$. Indeed, $\nabla z_{n} \rightarrow 0$ weakly in $L^{p(x)}\left(\Omega ; \mathbb{R}^{N}\right)$ then $A_{2, n} \rightarrow 0$. Similarly, since $\nabla z_{n} \chi_{\left\{\left|u_{n}\right|>k\right\}}=-\nabla T_{k}(u) \chi_{\left\{\left|u_{n}\right|>k\right\}} \rightarrow 0$ strongly in
$L^{p^{\prime}(x)}\left(\Omega ; \mathbb{R}^{N}\right)$, while $\left|\nabla u_{n}\right|^{p(x)-2} \nabla u_{n} \varphi^{\prime}\left(z_{n}\right) e^{\delta\left|u_{n}\right|} \psi \quad$ is bounded in $L^{p^{\prime}(x)}\left(\Omega ; \mathbb{R}^{N}\right)$, by (6), 20) and Remark 1 we obtain $A_{3, n} \rightarrow 0$. Therefore, we have proved that:

$$
A_{n}=A_{1, n}+o(1)
$$

For the integral $B_{n}$ while $\varphi\left(z_{n}\right)$ has the same sign as $c\left(u_{n}\right)$ on the set $\left\{\left|u_{n}\right|>k\right\}$ we have

$$
\begin{aligned}
B_{n}= & \int_{\left\{\left|u_{n}\right| \leq k\right\}} c\left(T_{k}\left(u_{n}\right)\right) \varphi\left(z_{n}\right) e^{\delta\left|T_{k}\left(u_{n}\right)\right|} \psi \\
& +\int_{\left\{\left|u_{n}\right|>k\right\}} c\left(u_{n}\right) \varphi\left(z_{n}\right) e^{\delta\left|u_{n}\right|} \psi \\
& \geq \int_{\left\{\left|u_{n}\right| \leq k\right\}} c\left(T_{k}\left(u_{n}\right)\right) \varphi\left(z_{n}\right) e^{\delta\left|T_{k}\left(u_{n}\right)\right|} \psi
\end{aligned}
$$

the last integrand converges pointwise and it is bounded then $\int_{\left\{\left|u_{n}\right| \leq k\right\}} c\left(T_{k}\left(u_{n}\right)\right) \varphi\left(z_{n}\right) e^{\delta\left|T_{k}\left(u_{n}\right)\right|} \psi$ goes to zero. Therefore, we obtain that:

$$
B_{n} \geq o(1)
$$

Let us examine $C_{n}$ and $D_{n}$ together. We first fix $\delta$ such that

$$
\delta>d
$$

Since $\varphi\left(z_{n}\right) \operatorname{sign}\left(u_{n}\right)=\left|\varphi\left(z_{n}\right)\right|$ on the set $\left\{\left|u_{n}\right|>k\right\}$ we have

$C_{n}+E_{n} \leq d \int_{\Omega}\left|\nabla u_{n}\right|^{p(x)}\left|\varphi\left(z_{n}\right)\right| e^{\delta\left|u_{n}\right|} \psi$

$-\delta \int_{\Omega}\left|\nabla u_{n}\right|^{p(x)} \varphi\left(z_{n}\right) e^{\delta\left|u_{n}\right|} \operatorname{sign}\left(u_{n}\right) \psi$

$\leq(d+\delta) \int_{\left\{\left|u_{n}\right| \leq k\right\}}\left|\nabla T_{k}\left(u_{n}\right)\right|^{p(x)}\left|\varphi\left(z_{n}\right)\right| e^{\delta\left|T_{k}\left(u_{n}\right)\right|} \psi$

$+(d-\delta) \int_{\left\{\left|u_{n}\right|>k\right\}}\left|\nabla u_{n}\right|^{p(x)} \varphi\left(z_{n}\right) e^{\delta\left|u_{n}\right|} \psi$

$\leq(d+\delta) \int_{\left\{\left|u_{n}\right| \leq k\right\}}\left|\nabla T_{k}\left(u_{n}\right)\right|^{p(x)}\left|\varphi\left(z_{n}\right)\right| e^{\delta\left|T_{k}\left(u_{n}\right)\right|} \psi$

$=(d+\delta) \int_{\left\{\left|u_{n}\right| \leq k\right\}}\left[\left|\nabla T_{k}\left(u_{n}\right)\right|^{p(x)-2} \nabla T_{k}\left(u_{n}\right)\right.$

$\left.-\left|\nabla T_{k}(u)\right|^{p(x)-2} \nabla T_{k}(u)\right] \nabla z_{n}\left|\varphi\left(z_{n}\right)\right| e^{\delta\left|T_{k}\left(u_{n}\right)\right|} \psi$

$+(d+\delta) \int_{\left\{\left|u_{n}\right| \leq k\right\}}\left|\nabla T_{k}\left(u_{n}\right)\right|^{p(x)-2} \nabla T_{k}\left(u_{n}\right) \nabla T_{k}(u) \mid$

$\varphi\left(z_{n}\right) \mid e^{\delta\left|T_{k}\left(u_{n}\right)\right|} \psi$

$+(d+\delta) \int_{\left\{\left|u_{n}\right| \leq k\right\}}\left|\nabla T_{k}(u)\right|^{p(x)-2} \nabla T_{k}(u) \nabla z_{n}\left|\varphi\left(z_{n}\right)\right| e^{\delta\left|T_{k}\left(u_{n}\right)\right|} \psi$

The last two integrals converge to zero.If we choose $\lambda$ such that:

$$
\lambda \geq 2(d+\delta)
$$

we have:

$$
(d+\delta)|\varphi(s)| \leq \frac{\varphi^{\prime}(s)}{2} \text { for every s in } \mathbb{R}
$$

then we can obtain:

$$
C_{n}+E_{n} \leq \frac{1}{2} A_{1, n}+o(1)
$$


Using Remark 1 we can observe that:

$$
D_{n} \rightarrow 0
$$

For the term $F_{n}$ we can see that $\left|\nabla \psi \| \varphi z_{n}\right|$ converge strongly to zero in $L^{r(x)}(\Omega)$ for every $r(x)>1$. by 20 the term $\left|\nabla u_{n}\right|^{p(x)-2} \nabla u_{n} e^{\delta\left|u_{n}\right|}$ is bounded in $L_{l o c}^{p^{\prime}(x)}(\Omega)$ then we have that:

$$
F_{n} \rightarrow 0
$$

For the term $G_{n}$ like before we have:

$$
\begin{aligned}
G_{n} & =\int_{\{|u| \leq k\}}|g|\left|\nabla z_{n}\right| \varphi^{\prime}\left(z_{n}\right) e^{\delta\left|u_{n}\right|} \psi \\
& +\int_{\{|u|>k\}}|g|\left|\nabla z_{n}\right| \varphi^{\prime}\left(z_{n}\right) e^{\delta\left|u_{n}\right|} \psi \\
& =G_{1, n}+G_{2, n}
\end{aligned}
$$

since

$$
|g| \varphi^{\prime}\left(z_{n}\right) e^{\delta\left|u_{n}\right|} \psi \chi_{\left\{\left|u_{n}\right| \leq k\right\}} \rightarrow|g| \varphi^{\prime}(0) e^{\delta\left|T_{k} u\right|} \psi \chi_{\{|u| \leq k\}}
$$

almost everywhere in $\Omega$ and

$$
|g| \varphi^{\prime}\left(z_{n}\right) e^{\delta\left|u_{n}\right|} \psi \chi_{\left\{\left|u_{n}\right| \leq k\right\}} \leq|g| \varphi^{\prime}(2 k) e^{\delta k} \psi
$$

Therefore by Lebesgue's theorem we have:

$$
|g| \varphi^{\prime}\left(z_{n}\right) e^{\delta\left|u_{n}\right|} \psi \chi_{\left\{\left|u_{n}\right| \leq k\right\}} \rightarrow|g| \varphi^{\prime}(0) e^{\delta\left|T_{k} u\right|} \psi \chi_{\{|u| \leq k\}}
$$

strongly in $L^{p^{\prime}(x)}(\Omega)$. Indeed, $\nabla z_{n} \rightarrow 0$ weakly in $L^{p(x)}\left(\Omega ; \mathbb{R}^{N}\right)$ then $G_{1, n} \rightarrow 0$. Similarly, since $\left|\nabla z_{n}\right| \chi_{\left\{\left|u_{n}\right|>k\right\}}=\left|\nabla T_{k}(u)\right| \chi_{\left\{\left|u_{n}\right|>k\right\}} \rightarrow 0$ strongly in $L^{p^{\prime}(x)}\left(\Omega ; \mathbb{R}^{N}\right)$, while $|g| \varphi^{\prime}\left(z_{n}\right) e^{\delta\left|u_{n}\right|} \psi$ is bounded in $L^{p^{\prime}(x)}\left(\Omega ; \mathbb{R}^{N}\right)$, by 20 and remark 1 we obtain $G_{2, n} \rightarrow 0$. Therefore, we have proved that:

$$
G_{n} \rightarrow 0
$$

Moreover

$$
\left|g \| \varphi\left(z_{n}\right)\right| \psi \rightarrow 0
$$

almost everywhere in $\Omega$ and

$$
\left|g\left\|\varphi\left(z_{n}\right)|\psi \leq| g\right\| \varphi(2 k)\right| \psi
$$

Therefore by Lebesgue's theorem we have:

$$
\left|g \| \varphi\left(z_{n}\right)\right| \psi \rightarrow 0
$$

strongly in $L^{p^{\prime}(x)}(\Omega)$. Indeed, $\nabla u_{n} e^{\delta\left|u_{n}\right|} \rightarrow \nabla u e^{\delta|u|}$ weakly in $L^{p(x)}\left(\Omega ; \mathbb{R}^{\mathbb{N}}\right)$, then:

$$
H_{n} \rightarrow 0
$$

Finally, $|\nabla \psi|\left|\varphi z_{n}\right|$ converge strongly to zero in $L^{r(x)}(\Omega)$ for every $r(x)>1$. by 20 the term $|g| e^{\delta\left|u_{n}\right|}$ is bounded in $L_{l o c}^{p^{\prime}(x)}(\Omega)$ then we have that:

$$
L_{n} \rightarrow 0
$$

Putting all inequalities 56), (57), (58, ,59, ,60, 61, 62, 63 and 64 we can conclude:

$$
A_{1, n} \rightarrow 0
$$

On the other hand we have

$$
\begin{gathered}
\int_{\left\{\left|u_{n}\right|>k\right\}}\left[\left|\nabla T_{k}\left(u_{n}\right)\right|^{p(x)-2} \nabla T_{k}\left(u_{n}\right)-\left|\nabla T_{k}(u)\right|^{p(x)-2} \nabla T_{k}(u)\right] \nabla z_{n} \\
\varphi^{\prime}\left(z_{n}\right) e^{\delta\left|T_{k}\left(u_{n}\right)\right|} \psi=\int_{\left\{\left|u_{n}\right|>k\right\}}\left|\nabla T_{k}(u)\right|^{p(x)} \varphi^{\prime}\left(k-T_{k}(u)\right) e^{\delta k} \psi \rightarrow 0
\end{gathered}
$$

From 65 and 66 we can conclude that:

$$
\begin{aligned}
& \int_{\Omega_{0}}\left[\left|\nabla T_{k}\left(u_{n}\right)\right|^{p(x)-2} \nabla T_{k}\left(u_{n}\right)-\left|\nabla T_{k}(u)\right|^{p(x)-2} \nabla T_{k}(u)\right] \\
& \left(\nabla T_{k}\left(u_{n}\right)-\nabla T_{k}(u)\right) \rightarrow 0
\end{aligned}
$$

Finally, using the Lemma 2 we have:

$$
\nabla T_{k}\left(u_{n}\right) \rightarrow \nabla T_{k}(u) \quad \text { strongly in } L^{p(x)}\left(\Omega_{0} ; \mathbb{R}^{\mathbb{N}}\right)
$$

\section{Step 3: End of the proof}

Observing that:

$$
\nabla u_{n}-\nabla u=\nabla T_{k} u_{n}-\nabla T_{k} u+\nabla G_{k} u_{n}-\nabla G_{k} u
$$

Let $\Omega_{0}$ be an open set compactly contained in $\Omega$, using (54) and 68 we have:

$$
\nabla u_{n} \rightarrow \nabla u \quad \text { strongly in } L^{p(x)}\left(\Omega_{0} ; \mathbb{R}^{\mathbb{N}}\right)
$$

To obtain (51) we have to pass to the limit in the distributional formulation of problem (5) using (69). Finally, statement 52 follows easily from Proposition 4 and 69, using Fatou's Lemma.

\section{Boundedness of solutions}

In this section we will gave some regularity on the solution of the problem (1) using an adaptation of a classical technique due to Stampacchia. To do this we need the following lemma (see [15]):

Lemma 4 Let $\phi$ be a non-negative, non-increasing function defined on the halfline $\left[k_{0}, \infty\right)$. Suppose that there exist positive constants $A, \mu, \beta$, with $\beta>1$, such that

$$
\phi(h) \leq \frac{A}{(h-k)^{\mu}} \phi(k)^{\beta}
$$

for every $h>k \geq k_{0}$. Then $\phi(k)=0$ for every $k \geq k_{1}$, where

$$
k_{1}=k_{0}+A^{1 / \mu} 2^{\beta /(\beta-1)} \phi\left(k_{0}\right)^{(\beta-1) / \mu}
$$

The result that we are going to prove is the following:

Theorem 2 Suppose that (3) holds. Then every solution $u$ of (1); which is specified in (4) is essentially bounded, and

$$
\left\|u_{n}\right\|_{L^{\infty}(\Omega)} \leq C
$$

The proof relies on the combined use of the wellknown technique by Stampacchia (see [15]) and suitable exponential test functions, as in [16]. 
Proof: Since 42 we can obtain an estimate for $\int_{\Omega}|u|^{p(x)-1} \varphi\left(G_{k}(u)\right)$ then for some constant $k_{0}=k(\lambda)$ sufficiently large we have

$$
\operatorname{meas}\left(A_{k_{0}}\right)<1
$$

where

$$
A_{k}=\{x \in \Omega:|u|>k\}
$$

as before we can take the test function $\varphi\left(G_{k}(u)\right)$ then we have:

$$
\begin{aligned}
& \int_{A_{k}}\left|\nabla G_{k}(u)\right|^{p(x)} \varphi^{\prime}\left(G_{k}(u)\right)+\alpha_{0} \int_{A_{k}}|u|^{p(x)-1}\left|\varphi\left(G_{k}(u)\right)\right|, \\
& \leq d \int_{A_{k}}\left|\nabla G_{k}(u)\right|^{p(x)}\left|\varphi\left(G_{k}(u)\right)\right|+\int_{A_{k} \cap\{|f|>1\}}\left|f \| \varphi\left(G_{k}(u)\right)\right| \\
& +\int_{A_{k} \cap\{|f| \leq 1\}}\left|\varphi\left(G_{k}(u)\right)\right|+\int_{A_{k}}\left|g \| \nabla G_{k}(u)\right| \varphi^{\prime}\left(G_{k}(u)\right)
\end{aligned}
$$

As in the proof of Proposition 4 one has:

$$
\begin{aligned}
& \int_{A_{k}}\left|g \| \nabla G_{k}(u)\right| \varphi^{\prime}\left(G_{k}(u)\right) \\
& \leq \frac{1}{4} \int_{A_{k}}\left|\nabla G_{k}(u)\right|^{p(x)} \varphi^{\prime}\left(G_{k}(u)\right)+C_{22} \int_{A_{k}}|g|^{p^{\prime}(x)} \varphi^{\prime}\left(G_{k}(u)\right)
\end{aligned}
$$

and if $\lambda \geq 4 d$ and $k \geq k_{0}(\lambda)$ (large enough) where

$$
\alpha_{0} k_{0}^{p_{-}-1} \geq 4
$$

then

$\frac{1}{2} \int_{A_{k}}\left|\nabla G_{k}(u)\right|^{p(x)} \varphi^{\prime}\left(G_{k}(u)\right)+\frac{3 \alpha_{0}}{4} \int_{A_{k}}|u|^{p(x)-1}\left|\varphi\left(G_{k}(u)\right)\right|$,

$\leq \int_{\left(A_{k} \backslash A_{k+1}\right) \cap\{|f|>1\}}\left|f \| \varphi\left(G_{k}(u)\right)\right|$

$+\int_{A_{k+1} \cap\{|f|>1\}}\left|f \| \varphi\left(G_{k}(u)\right)\right|+C_{22} \varphi^{\prime}(1) \int_{A_{k} \backslash A_{k+1}}|g|^{p^{\prime}(x)}$

$+C_{22} \int_{A_{k+1}}|g|^{p^{\prime}(x)} \varphi^{\prime}\left(G_{k}(u)\right)$

using Hölder inequality we have:

$$
\begin{aligned}
& \int_{\left(A_{k} \backslash A_{k+1}\right) \cap\{|f|>1\}}\left|f \| \varphi\left(G_{k}(u)\right)\right| \\
& \leq \varphi(1)\left(\frac{1}{q_{-}}+\frac{1}{q_{-}^{\prime}}\right)\|f\|_{L^{q(x)}(\{|f|>1\})}\left(\operatorname{meas}\left(A_{k}\right)\right)^{\frac{1}{q_{+}^{\prime}}}
\end{aligned}
$$

by Hölder's inequality and interpolation we obtain:

$$
\int_{A_{k+1} \cap\{|f|>1\}}\left|f \| \varphi\left(G_{k}(u)\right)\right|
$$

$\leq\|f\|_{L^{q_{-}}\left(A_{k+1} \cap\{|f|>1\}\right)}\left\|\varphi\left(G_{k}(u)\right)\right\|_{L^{p *-}-p_{-}\left(A_{k+1}\right)}^{\frac{N}{p-q_{-}}}\left\|\varphi\left(G_{k}(u)\right)\right\|_{L^{1}\left(A_{k+1}\right)}^{1-\frac{N}{p_{-}}}$

while 28), 71 and using Young's and sobolev's in- equalities we can deduce that:

$$
\begin{aligned}
& \int_{A_{k+1} \cap\{|f|>1\}}\left|f \| \varphi\left(G_{k}(u)\right)\right| \\
& \leq \frac{1}{8}\left\|\nabla \psi\left(G_{k}(u)\right)\right\|_{L^{p(x)}\left(A_{k}\right)}^{p_{-}} \\
& +C_{23}\|f\|_{\left.L^{q-(}(|| f \mid>1\}\right)}^{\frac{p_{-q}-q_{-}}{p-q_{0}}}\left\|\varphi\left(G_{k}(u)\right)\right\|_{L^{1}\left(A_{k}\right)} \\
& \leq \frac{1}{8} \int_{A_{k}}\left|\nabla \psi\left(G_{k}(u)\right)\right|^{p(x)}+1 d x \\
& +C_{23}\|f\|_{\left.L^{q-}(|| f \mid>1\}\right)}^{\frac{p-q_{-}}{p-q_{-}}}\left\|\varphi\left(G_{k}(u)\right)\right\|_{L^{1}\left(A_{k}\right)} \\
& \leq \frac{1}{8} \int_{A_{k}}\left|\nabla\left(G_{k}(u)\right)\right|^{p(x)} \varphi^{\prime}\left(G_{k}(u)\right) d x+\frac{1}{8} \operatorname{meas}\left(A_{k}\right) \\
& +C_{23}\|f\|_{L^{q-}(\{|f|>1\})}^{\frac{p_{-q}-N}{p-1}}\left\|\varphi\left(G_{k}(u)\right)\right\|_{L^{1}\left(A_{k}\right)}
\end{aligned}
$$

Therfore, choosing $k_{0}$ such that:

$$
C_{23}\|f\|_{\left.L^{q-(}(|| f \mid>1\}\right)}^{\frac{p_{-q}-q_{-}-N}{p-N}} \leq \frac{\alpha_{0} k_{0}^{p_{-}-1}}{4}
$$

the second integral in the right-hand side of 74 can be absorbed by the left-hand side.

In view of Hölder's inequality and (71) and (3) we have:

$$
\begin{aligned}
& C_{22} \varphi^{\prime}(1) \int_{A_{k} \backslash A_{k+1}}|g|^{p^{\prime}(x)} \\
& \leq C_{23}\left(\int_{A_{k}}|g|^{p^{\prime}(x)}\right)^{\eta}\left(\operatorname{meas}\left(A_{k}\right)\right)^{1-\frac{p_{+}^{\prime}}{r_{-}}} \\
& \leq C_{24}\left(\|g\|_{L^{r(x)}\left(A_{k}\right)}\right)^{\delta^{\prime \prime}}\left(\operatorname{meas}\left(A_{k}\right)\right)^{1-\frac{p_{+}^{\prime}}{r_{-}}}
\end{aligned}
$$

where

$$
\begin{gathered}
\eta= \begin{cases}\frac{p_{-}^{\prime}}{r_{+}} & \text {if } \int_{A_{k}}|g|^{p^{\prime}(x)} \leq 1, \\
\frac{p_{+}^{\prime}}{r_{-}} & \text {if } \int_{A_{k}}|g|^{p^{\prime}(x)}>1 .\end{cases} \\
\delta^{\prime \prime}= \begin{cases}\frac{\eta}{r_{-}} & \text {if }\|g\|_{L^{r(x)}\left(A_{k}\right)} \geq 1, \\
\frac{\eta}{r_{+}} & \text {if }\|g\|_{L^{r(x)}\left(A_{k}\right)}<1 .\end{cases}
\end{gathered}
$$

Finally, with similar calculations, using (39) we have:

$$
\begin{aligned}
& C_{22} \int_{A_{k+1}}|g|^{p^{\prime}(x)} \varphi^{\prime}\left(G_{k}(u)\right) \\
& \leq C_{24} \int_{A_{k+1} \cap\{|g|>1\}}|g|^{p^{\prime}(x)}\left|\varphi\left(G_{k}(u)\right)\right| \\
& +C_{24} \int_{A_{k+1} \cap\{|g| \leq 1\}}\left|\varphi\left(G_{k}(u)\right)\right|
\end{aligned}
$$

If we choose $k_{0}$ such that:

$$
\alpha_{0} k_{0}^{p_{-}-1}>4 C_{24}
$$

then

$$
\begin{aligned}
& C_{22} \int_{A_{k+1}}|g|^{p^{\prime}(x)} \varphi^{\prime}\left(G_{k}(u)\right) \\
& \leq C_{24} \int_{A_{k+1} \cap\{|g|>1\}}|g|^{p_{+}^{\prime}}\left|\varphi\left(G_{k}(u)\right)\right| \\
& +\frac{\alpha_{0}}{4} \int_{A_{k}}|u|^{p(x)-1}\left|\varphi\left(G_{k}(u)\right)\right|
\end{aligned}
$$


by Hölder's inequality and interpolation we obtain:

$C_{22} \int_{A_{k+1}}|g|^{p^{\prime}(x)} \varphi^{\prime}\left(G_{k}(u)\right)$

$\leq C_{24}\|g\|_{L^{r_{-}}\left(\Omega, \mathbb{R}^{N}\right)}^{p_{+}^{\prime}}\left\|\varphi\left(G_{k}(u)\right)\right\|_{L^{p_{-}-1 p_{-}}\left(A_{k}\right)}^{\frac{p_{+}^{\prime} N}{p_{-}}}\left\|\varphi\left(G_{k}(u)\right)\right\|_{L^{1}\left(A_{k}\right)}^{1-\frac{p_{+}^{\prime} N}{p_{-} r_{-}}}$

$+\frac{\alpha_{0}}{4} \int_{A_{k}}|u|^{p(x)-1}\left|\varphi\left(G_{k}(u)\right)\right|$

as before while 28, , 71 and using Young's and sobolev's inequalities we can deduce that:

$$
\begin{aligned}
& C_{22} \int_{A_{k+1}}|g|^{p^{\prime}(x)} \varphi^{\prime}\left(G_{k}(u)\right) \\
& \leq \frac{1}{8} \int_{A_{k}}\left|\nabla\left(G_{k}(u)\right)\right|^{p(x)} \varphi^{\prime}\left(G_{k}(u)\right) d x+\frac{1}{8} \operatorname{meas}\left(A_{k}\right) \\
& +C_{25}\|g\|_{L^{r}-\left(\Omega, \mathbb{R}^{N}\right)}^{\frac{p_{+}^{\prime} p_{-} r_{-}}{p_{-} p_{+}^{\prime}}}\left\|\varphi\left(G_{k}(u)\right)\right\|_{L^{1}\left(A_{k}\right)} \\
& +\frac{\alpha_{0}}{4} \int_{A_{k}}|u|^{p(x)-1}\left|\varphi\left(G_{k}(u)\right)\right|
\end{aligned}
$$

Therefore, by taking $k_{0}$ satistying (71), (73), (75, , 76) and the further condition:

$$
C_{25}\|g\|_{L^{r_{-}}\left(\Omega, \mathbb{R}^{N}\right)}^{\frac{p_{-}^{\prime} p_{-} r_{-}}{p_{-}-p_{+}^{\prime} N}} \leq \frac{\alpha_{0} k_{0}^{p_{-}-1}}{4}
$$

one obtains, for every $k \geq k_{0}$ :

$$
\begin{aligned}
& \frac{1}{4} \int_{A_{k}}\left|\nabla G_{k}(u)\right|^{p(x)} \varphi^{\prime}\left(G_{k}(u)\right) \\
& \leq \varphi(1)\left(\frac{1}{q_{-}}+\frac{1}{q_{-}^{\prime}}\right)\|f\|_{L^{q(x)}(\{|f|>1\})}\left(\text { meas }\left(A_{k}\right)\right)^{\frac{1}{q_{+}^{\prime}}}+\frac{1}{4} \text { meas }\left(A_{k}\right)
\end{aligned}
$$

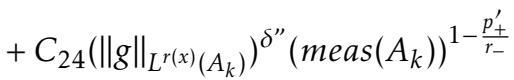

$$
\begin{aligned}
& \leq C_{26}\left(\text { meas }\left(A_{k}\right)\right)^{m}
\end{aligned}
$$

where $m=\min \left(\frac{1}{q_{+}^{\prime}}, 1-\frac{p_{+}^{\prime}}{r_{-}}\right)$in view of 26] and Sobolev's inequality we can obtain:

$$
\begin{aligned}
\left(\int_{A_{k}}\left|\psi\left(G_{k}(u)\right)\right|^{p *(x)}\right)^{\beta} & \leq\left\|\psi\left(G_{k}(u)\right)\right\|_{L^{p *(x)}\left(A_{k}\right)} \\
& \leq C_{27}\left(\text { meas }\left(A_{k}\right)\right)^{\frac{m}{\alpha}}
\end{aligned}
$$

Where:

$$
\begin{aligned}
& \alpha= \begin{cases}p_{+} & \text {if }\left\|\nabla \psi\left(G_{k}(u)\right)\right\|_{L^{p(x)}\left(A_{k}\right)} \leq 1, \\
p_{-} & \text {if }\left\|\nabla \psi\left(G_{k}(u)\right)\right\|_{L^{p(x)}\left(A_{k}\right)}>1 .\end{cases} \\
& \beta= \begin{cases}\frac{N-p_{-}}{N p_{-}} & \text {if }\left\|\psi\left(G_{k}(u)\right)\right\|_{L^{p *(x)}\left(A_{k}\right)} \leq 1, \\
\frac{N-p_{+}}{N p_{+}} & \text {if }\left\|\psi\left(G_{k}(u)\right)\right\|_{L^{p *(x)}\left(A_{k}\right)}>1 .\end{cases}
\end{aligned}
$$

We now take $h-k>1$ and recall that there exists $C_{28}\left(\lambda, p_{+}, p_{-}\right)$such that $|\psi(s)| \geq C_{28}|s|$ for every $s \in \mathbb{R}$ so that

$$
\begin{aligned}
{\left[C_{28}(h-k)\right]^{p_{-}} \text {meas }\left(A_{h}\right) } & \leq \int_{A_{h}} \mid \psi\left(G_{k}(u)\right)^{p *(x)} \\
& \leq \int_{A_{k}} \mid \psi\left(G_{k}(u)\right)^{p^{* *(x)}} \\
& \leq C_{29}\left(\text { meas }\left(A_{k}\right)\right)^{\frac{m}{\alpha \beta}}
\end{aligned}
$$

Then it follows for every $\mathrm{h}$ and $\mathrm{k}$ (such that $h>k \geq k_{0}$ ) that

$$
\text { meas }\left(A_{h}\right) \leq \frac{C_{30}}{[h-k]^{p_{-}}}\left(\text {meas }\left(A_{k}\right)\right)^{\frac{m}{\alpha \beta}}
$$

Since by (3), $\frac{m}{\alpha \beta}>1$ Lemma 4 applied to the function $\phi(h)=$ meas $\left(A_{h}\right)$ gives:

$$
\left\|u_{n}\right\|_{L^{\infty}(\Omega)} \leq C
$$

\section{References}

1. Y. Chen, S. Levine, M. Rao,, Variable exponent, linear growth functionals in image restoration., SIAM J. Appl. Math., 66, 1383-1406 (2006).

2. V. V. Zhikov, Averaging of functionals of the calculus of variations and elasticity theory., Math. USSR Izvestiya, 29(1), 33-66 (1987).

3. E. Azroul, H. Hjiaj, A. Touzani, Existence and regularity of entropy solutions for strongly nonlinear $p(x)$ - elliptic equations, Electronic Journal of Differential Equations, Vol. (2013), No. 68 , pp. 1-27.

4. M. B. Benboubker,H. Chrayteh, M. El Moumni, H. Hjiaj; Entropy and Renormalized Solutions for Nonlinear Elliptic Problem Involving Variable Exponent and Measure Data, Acta Mathematica Sinica, English Series Jan., 2015, Vol. 31, No. 1, pp. 151-169.

5. C. Yazough, E. Azroul, H. Redwane; Existence of solutions for some nonlinear elliptic unilateral problems with measure data, Electronic Journal of Qualitative Theory of Diferential Equations 2013, No. 43, 1-21;

6. Q. Zhang; Existence of radial solutions for $p(x)$-Laplacian equations in $\mathbb{R}^{N}$, J. Math. Anal. Appl. 315 (2006) 506-516.

7. A. Dall'Aglio D. Giachetti J.-P. Puel, Nonlinear elliptic equations with natural growth in general domains, Annali di Matematica 181, 407-426 (2002).

8. V. Ferone, F. Murat; Nonlinear problems having natural growth in the gradient: an existence result when the source terms are small, Nonlinear Analysis 42 (2000) 1309-1326.

9. Guowei Dai; Infinitely many solutions for a $p(x)$-Laplacian equation in $\mathbb{R}^{N}$, Nonlinear Analysis 71 (2009) 1133-1139;

10. X. L. Fan, D. Zhao; On the generalised Orlicz-Sobolev Space $W^{k, p(x)}(\Omega)$, J. Gansu Educ. College 12(1) (1998), 1-6.

11. D. Zhao, W. J. Qiang, X. L. Fan; On generalized Orlicz spaces $L^{p(x)}(\Omega)$, J. Gansu Sci. 9(2), 1997, 1-7.

12. P. Harjulehto, P. Hästö; Sobolev Inequalities for Variable Exponents Attaining the Values 1 and n, Publ. Mat. 52 (2008), no. 2, 347-363.

13. L. Diening, P. Harjulehto, P. Hästö, M. Ržička; Lebesgue and Sobolev Spaces with Variable Exponents, vol. 2017 of Lecture Notes in Mathematics, Springer, Heidelberg, Germany, 2011.

14. J. L. Lions; Quelques methodes de résolution des problèmes aux limites non linéaires, Dunod et Gauthiers-Villars, Paris 1969.

15. G. Stampacchia; Equations elliptiques du second ordre à coefficients discontinus, Séminaire de Mathématiques Suprieures. No. 16, Montréal, Que.: Les Presses de l'Université de Montréal (1966)

16. L. Boccardo, F. Murat, J.-P. Puel,; $L^{\infty}$ estimate for some nonlinear elliptic partial differential equations and application to an existence result,SIAM J. Math. Anal. (2) 23, 326-333 (1992) 